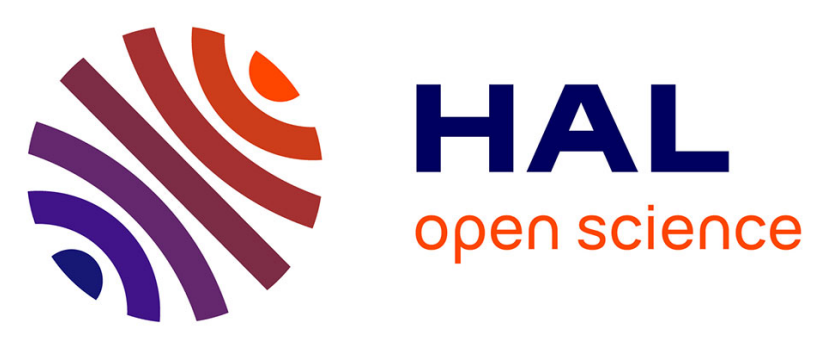

\title{
L'écriture épique ovidienne face à 'sa' tradition : représentation et mise à distance de la poétique de l'Enéide dans les Métamorphoses
}

Florence Klein

\section{- To cite this version:}

Florence Klein. L'écriture épique ovidienne face à 'sa' tradition: représentation et mise à distance de la poétique de l'Enéide dans les Métamorphoses. Aline Estèves, Jean Meyers. Tradition et innovation dans l'épopée latine, de l'Antiquité au Moyen-Âge, Ausonius Editions, pp.63-80, 2014, 978-2-35613120-1. hal-01302205

\section{HAL Id: hal-01302205 \\ https://hal.science/hal-01302205}

Submitted on 13 Apr 2016

HAL is a multi-disciplinary open access archive for the deposit and dissemination of scientific research documents, whether they are published or not. The documents may come from teaching and research institutions in France or abroad, or from public or private research centers.
L'archive ouverte pluridisciplinaire HAL, est destinée au dépôt et à la diffusion de documents scientifiques de niveau recherche, publiés ou non, émanant des établissements d'enseignement et de recherche français ou étrangers, des laboratoires publics ou privés. 


\section{L'écriture épique ovidienne face à 'sa' tradition : représentation et mise à distance de la poétique de l'Énéide dans les Métamorphoses.}

Dans le cadre d'un questionnement d'ensemble portant sur « la tradition et l'innovation dans l'épopée latine », nous nous intéresserons ici au rapport étroit et complexe qu'entretient la singulière épopée ovidienne avec le modèle qu'elle désigne comme 'sa' propre tradition, à savoir l'Énéide. Il est en effet bien connu que le poème de Virgile, pour ainsi dire immédiatement élevé par ses contemporains au rang de classique, a suscité un positionnement générique fort de la part de son premier successeur, celui que l'on a appelé son «meilleur lecteur $»^{1}-$ ce qui ne signifie pas qu'il en ait été le plus respectueux.

La mise en valeur de l'adjectif possessif dans mon titre correspond à la réévaluation de la notion de tradition littéraire qui a été élaborée dans les années 1990 par Alain Deremetz ${ }^{2}$ et Stephen Hinds ${ }^{3}$ : ces deux chercheurs ont en effet montré qu'il ne fallait pas envisager cette dernière comme une donnée objective et immuable (l'œuvre ultérieure étant supposée être le réceptacle passif de l'œuvre antérieure) mais en tant que cette tradition est - pourrait-on dire le 'produit' des œuvres ultérieures. De fait, elle est à chaque fois refaçonnée par les œuvres qui la convoquent : ce sont elles qui font émerger, chez le(s) prédécesseur(s) par rapport $\mathrm{au}(\mathrm{x})$ quel(s) elles se situent, les éléments qui leur permettent d'en en faire leur modèle ou leur contre-modèle, et éventuellement les deux à la fois.

En l'occurrence, la présentation que fait Ovide d'un Virgile exhibé à la fois comme modèle (par certains aspects) et comme contre-modèle de son épopée se caractérise par l'opposition entre deux 'avatars virgiliens' co-présents dans le texte ovidien. Nous verrons en effet que, d'un côté, pour mieux se distinguer de lui et apparaître d'emblée comme une alternative à Virgile, Ovide accentue à dessein leur altérité et, pour cela, présente dans son propre texte une image radicalisée de 'son' contre-modèle virgilien, tandis que, d'un autre côté, il s'efforce de pointer rétrospectivement dans l'Énéide ce qu'on pourrait appeler des tentations conjurées, des moments où Virgile aurait esquissé la possibilité d'un autre modèle, sitôt refusé et dépassé, et qu'Ovide, quant à lui, développerait pleinement.

Autrement dit, tandis que l'auteur des Métamorphoses (re)construit dans son œuvre l'image d'un Virgile radicalement 'autre' (que lui-même), un « anti-Ovide » avant la lettre ${ }^{4}$, il montre

\footnotetext{
${ }^{1}$ O’Hara 1995-1996.

${ }^{2}$ Deremetz 1995, 113-114.

${ }^{3}$ Hinds 1997

${ }^{4}$ Dans le même ordre d'idée - mais à propos de la vision traditionnellement transmise du rapport à Auguste des deux poètes -, on rappellera la suggestion de Tarrant 2002, 26-27, se demandant si Ovide, en se définissant lui-
} 
également ce qu'aurait pu faire Virgile s'il avait suivi ces tentations alternatives auxquelles lui même s'adonne à plein 5 .

En même temps, ce positionnement pour le moins complexe des Métamorphoses par rapport à l'Énéide a une valeur totalisante, puisqu'il engage la question qui est au cœur du genre épique, celle de l'ordre et du désordre - une problématique à la fois idéologique et poétique, ces deux aspects étant indissociables dans un genre qui lie profondément forme narrative et discours idéologique et, pour cela, repose aussi sur une certaine organisation du monde, une cosmologie épique qui sera mon point de départ.

\section{Le cosmos épique dans l'Énéide et dans les Métamorphoses}

Avant d'aborder la question de l'organisation du cosmos dans les épopées virgilienne et ovidienne, du moins sur les points précis par lesquelles ces deux représentations de l'univers épique se distinguent l'une de l'autre, il nous faut revenir brièvement sur l'emploi délicat des notions d'ordre et de désordre et/ou de chaos à propos des Métamorphoses. En effet, l'opposition entre ordre et désordre est naturellement invoquée par la critique lorsqu'est abordée la cosmogonie ovidienne ainsi que ce dont elle offre l'image, que ce soit la représentation du pouvoir politique ou celle de l'ordonnancement poétique de l'œuvre. Le lien puissant qui unit ces deux derniers aspects est d'ailleurs illustré dans le texte lui-même, au livre 6, avec le concours entre Pallas-Minerve et Arachné dont les tapisseries respectives traduisent une vision du monde et du pouvoir divin inséparable de leur esthétique, l'œuvre de Pallas étant régi par un principe d'ordre, celle d'Arachné par un apparent désordre ${ }^{6}$.

Les Métamorphoses s'ouvrent sur la présentation initiale de l'univers comme un chaos informe qu'un démiurge, comparé à un artiste ${ }^{7}$, transforme en cosmos organisé ${ }^{8}$ : ce dernier met en forme cette masse pesante et inerte, en dégageant - nous allons y revenir - les éléments les plus légers auxquels il confère une place éminente, et en laissant retomber en bas le poids de la matière brute, qui n'a pas été travaillée. Comment comprendre, en relation avec

\footnotetext{
même par opposition à Virgile sur ce point, n'a pas fortement contribué à créer l'image figée d'un Virgile courtisan que la critique récente s'attache désormais à nuancer.

${ }^{5}$ Stephen Hinds a ainsi, par exemple, illustré la manière dont Ovide reconstruit rétrospectivement l'image de Virgile comme celle d'un «précurseur hésitant des Métamorphoses» à propos ce que l'on appelle traditionnellement l' «Énéide ovidienne » en Met. 13-14 : Hinds 1998, 106.

${ }^{6}$ Sur cet épisode voir, entre autres études, Leach 1974, Sauron 1982, Feeney 1991, 191-2, Fabre-Serris 1995 , 301-311. En particulier, Hardie 2009, 85-86 suggère que l'œuvre de Minerve représente précisément 1'Enéide, tandis que celle d'Arachné - comme cela a été plus souvent commenté - représente les Métamorphoses ellesmêmes : en ce cas, Ovide intègrerait ici aussi, dans les Métamorphoses, une représentation de son contre-modèle qu'il radicalise dans son altérité afin de mieux s'en distinguer.

${ }^{7}$ Sur l'importance de ce choix ovidien d'une création démiurgique et non spontanée, voir Wheeler 1995.

${ }^{8}$ Ov., Met. 1.5-75.
} 
la vision du monde et du poème qui sous-tend tout le reste du texte ovidien, cette première évocation d'un chaos négativement présenté et remplacé, grâce à l'œuvre d'un démiurge bienfaiteur, par un cosmos organisé ?

Dans ce début, certains critiques ont voulu voir l'image en miniature du mouvement général qui conduirait les Métamorphoses du chaos à la célébration de l'ordre instauré par Auguste au livre 15 , selon une lecture de l'œuvre qui l'apparenterait au modèle «minervéen » ${ }^{9}$. Les analyses les plus récentes insistent généralement, au contraire, sur le fait que cet ordre du cosmos est aussitôt et constamment démenti dans une œuvre où règnent le changement, les bouleversements cosmiques et les métamorphoses ${ }^{10}$ : dans la perspective d'une lecture « arachnéenne » du poème, cette description initiale d'un cosmos organisé se justifierait alors surtout par le contraste qu'elle permet avec le retour au chaos, c'est-à-dire au mélange et au brouillage des frontières entre les éléments, qui dominerait dans l'œuvre ${ }^{11}$.

Tout en adhérant à cette lecture «arachnéenne », je voudrais cependant la nuancer en revenant sur l'hypothèse d'un cosmos ordonné qui serait là uniquement pour mettre en valeur le désordre qui lui succède comme son contraire. Car force est de constater, d'une part, que cette cosmogonie est positivement présentée dans l'œuvre et surtout que son contraire, le chaos initial, ne l'est pas et, d'autre part, que si l'œuvre se caractérise ensuite par le désordre et l'incessant mélange des éléments, il ne s'agit en rien d'un retour au chaos qui n'était qu'une matière uniforme et brute, une masse pesante, informe et inerte. Il semble bien plutôt que l'esthétique « arachnéenne » du mouvement et de l'apparent désordre qui régit l'ensemble de l'œuvre, loin de s'opposer à ce cosmos organisé par le démiurge, est au contraire permise et même programmée par ce dernier.

Pour cela, il est important de noter qu'Ovide a fait bouger les termes en présence par rapport à son illustre prédécesseur : si le monde représenté par Arachné (qui, sans être ordonné, ne s'apparente pas pour autant au chaos conçu comme amas uniforme d'éléments indifférenciés)

\footnotetext{
${ }^{9}$ Pour une lecture 'augustéenne' de la cosmologie, voir Maurach 1979, 134-140. L'adoption d'une perspective 'minervéenne' sur le poème des Métamorphoses est, par exemple, illustrée par Otis 1970.

${ }^{10}$ Voir, par ex., Hardie 1993 qui note que le mouvement de l'œuvre ovidienne est à l'inverse de celui de l'Énéide qui s'ouvre sur une image de chaos (avec la tempête initiale) et tend vers une stabilité qu'il faut rétablir quand elle est menacée. Chez Ovide, au contraire, avec la cosmogonie initiale, l'ordre et la paix semblent instaurés dès le début par le démiurge, mais sont immédiatement et constamment bouleversés : «In Virgilian terms, the act of creation represents the stability that is the epic's goal and which in the epic seems to have been reached when we are scarcely under way. The rest of the poem is an account of the transformations that continually dissolve the god's perfect handiwork ; a poem on change has a vested interest in the repetition of chaos, in things that do not keep their shape », écrit $\mathrm{Ph}$. Hardie (p. 61). La nuance que je me proposerai alors d'apporter ici est de considérer que, pour en arriver là, Ovide ne définit précisément pas son cosmos « en termes virgiliens », mais redonne, pour sa part, un sens différent aux termes de cette opposition virgilienne entre ordre et chaos.

${ }^{11}$ Pour diverses interprétations de ce contraste apparent entre l'ordre initial du cosmos et la suite du poème faite de changements et de désordres, voir par ex. Coleman 1971, 462 et Feeney 1991, 189, ou Wheeler 1999, 30-33.
} 
s'oppose à celui de Minerve, c'est que l'ordre représenté par cette dernière n'est précisément pas celui du cosmos créé par le démiurge ovidien, mais ressemble plutôt à un ordre du monde qui se voudrait 'virgilien', en tant notamment qu'il est régi par des dieux que caractérise une «auguste gravité » (augusta grauitas) ${ }^{12}$ dont nous montrerons à quel point elle est inappropriée dans le cosmos ovidien, tel que le démiurge l'a façonné.

Autrement dit, il ne s'agit pas tant pour Ovide de 'prendre le parti du chaos' contre un cosmos bien ordonné tel qu'il est mis en avant chez Virgile, que de redéfinir autrement, et contre ce dernier, ces notions d'ordre, de cosmos et de chaos. De fait, à un chaos négativement présenté (et assimilé à une masse informe à laquelle l'univers ne sera plus jamais réduit, même dans les moments de plus grand désordre cosmique) s'oppose d'emblée le cosmos ovidien qu'a créé le démiurge et qui contient en lui la possibilité de tous les désordres qui suivront dans l'œuvre. Pour comprendre la valorisation du cosmos façonné par l'art du démiurge, il nous faut alors voir en quoi ce cosmos ovidien (qui autorise voire programme ce qu'un Virgile, pour sa part, qualifierait de chaos) se distingue du cosmos virgilien. Qu'est-ce qui, au cœur même de cet ordre initial, permet déjà les changements et les désordres à venir ?

La réponse me paraît résider dans les deux vers de la cosmogonie ovidienne qui suivent immédiatement la description des vents exerçant leurs forces contraires dans le ciel : au dessus de ces derniers, écrit Ovide, le démiurge «posa l'éther limpide et privé de toute pesanteur (grauitate carentem), pur de toute la lie terrestre $»^{13}$. En cela, le cosmos des Métamorphoses se distingue de celui de l'Énéide, et cette divergence est ainsi commentée par par Denis Feeney :

\footnotetext{
«The oddness of the control is caught in a moment of comparison with Vergil's universe: Vergil's Jupiter controls the winds by putting on top of them a mass of high mountains (Aen.1.61) while Ovid's mundi fabricator places above them the aether, explicitely 'liquid and lacking weight, containing nothing of earthly sediment'». ${ }^{14}$
}

Tandis donc, note Denis Feeney, que le Jupiter virgilien contrôle les vents en les écrasant sous de lourdes montagnes (cela est dit au livre 1 de l'Énéide, nous y reviendrons), le démiurge ovidien n'a placé sur ces derniers que l'éther sans poids. Cette correction me semble fondamentale en ce qu'elle concerne le principe même qui régit chacun des deux cosmos d'un côté, le cosmos virgilien dont l'ordre est garanti par une forme de grauitas, qui réprime

\footnotetext{
${ }^{12}$ Ov. Met. 6.73 .

${ }^{13}$ Ov., Met. 1.67-8 : Haec super inposuit liquidum et grauitate carentem / aethera nec quicquam terrenae faecis habentem.

${ }^{14}$ Feeney 1991, 190.
} 
les éléments de désordre potentiel; de l'autre, au contraire, un cosmos ovidien régi par une substance sans poids, un principe de leuitas qui, au cœur de cette organisation ordonnée, permet les désordre futurs.

Avant de revenir plus précisément sur cette différence, il nous faut tout d'abord replacer ces deux vers dans le contexte général de la cosmogonie ovidienne. L'univers créé par le démiurge est, comme nous l'avons rappelé, organisé en une disposition hiérarchique des éléments en fonction de leur poids, la position supérieure étant réservée aux éléments les plus légers :

Ignea conuexi uis et sine pondere caeli emicuit summaque locum sibi fecit in arce ; proximus est aer illi leuitate locoque densior his tellus elementaque grandia traxit et pressa est grauitate sui...

L'essence ignée et sans poids de la voûte céleste s'élança et s'installa dans les hauteurs suprêmes ; le second après elle, par la légèreté et par le lieu, est l'air. Plus dense que ces derniers, la terre attira avec elle les éléments massifs et se tassa sous son propre poids. ${ }^{15}$

C'est ainsi que l'éther «sans poids » (sine pondere) s'élève dans les plus hautes régions du cosmos. On peut d'emblée relever la désignation summa arce pour désigner le lieu où se situe l'éther sans poids dans le cosmos - j'y reviendrai tout à l'heure. Un peu plus loin, ce critère du poids pour la disposition respective des éléments est rappelé lorsque le poète décrit la région de l'air ${ }^{16}$. Il est significatif que soit à nouveau soulignée, précisément ici, la position supérieure des éléments les plus légers dans l'organisation du cosmos: le fait que l'air, naturellement moins pesant que les autres éléments qu'il surplombe, soit lui-même dominé par un élément encore plus léger constitue - on le verra - une divergence fondamentale par rapport au modèle de l'Énéide. En effet, ce passage est directement suivi par la description des vents qui habitent la région de l'air et dont le poète précise qu'ils représentent un risque perpétuel de désordre cosmique, tant est grande la discorde entre eux ${ }^{17}$. Or cette évocation ovidienne des vents qui séjournent dans l'air est une variation sur le passage du premier livre de l'Énéide qui décrit la cave où sont enfermés ces mêmes vents :

... Hic uasto rex Aeolus antro

luctantes uentos tempestatesque sonoras imperio premit ac uinclis et carcere frenat.

\footnotetext{
${ }^{15}$ Ov., Met. $1.26-30$

${ }^{16} \mathrm{Ov} .$, Met. 1.52-53: Inminet his aer ; qui, quanto est pondere terrae / pondere aquae leuior, tanto est onerosior igni, «Suspendu au-dessus de ces dernières, il y a l'air : autant il est plus léger que le poids de la terre, que le poids de l'eau, autant il l'est moins que le feu »

${ }^{17}$ Ov., Met. 1.54-66.
} 


\begin{abstract}
Illi indignantes magno cum murmure montis circum claustra fremunt; celsa sedet Aeolus arce sceptra tenens, mollitque animos et temperat iras. Ni faciat, maria ac terras caelumque profundum quippe ferant rapidi secum uerrantque per auras. Sed pater omnipotens speluncis abdidit atris hoc metuens, molemque et montis insuper altos imposuit, regemque dedit, qui foedere certo et premere et laxas sciret dare iussus habenas.
\end{abstract}

Là, dans une vaste caverne, le roi Éole contient sous son pouvoir et réfrène, par les chaînes et le cachot, les vents en lutte et les tempêtes sonores. Eux, indignés, faisant résonner un immense murmure dans la montagne, frémissent près des portes fermées. En haut, Éole siège sur le sommet, son sceptre à la main; il apaise leurs élans et modère leurs colères. S'il ne le faisait pas, assurément, les mers, les terres et le ciel profond, ces vents les emporteraient violemment avec eux et les balaieraient dans les airs. Mais le père tout-puissant, qui craignait cela, les relégua dans de sombres cavernes et posa sur eux une masse de hautes montagnes, puis il leur donna un roi qui, conformément à un pacte précis, saurait, sur ses ordres, leur serrer ou leur relâcher les rênes. ${ }^{18}$

Virgile y mentionne la force potentiellement destructrice des vents, et précise que Jupiter, craignant celle-ci, les a placés sous le contrôle d'un roi, Éole, qui les maintient sous son pouvoir (imperio premit); en outre, pour les empêcher de ravager l'univers, le dieu a placé sur eux une lourde masse de hautes montagnes :

hoc metuens, molemque et montis insuper altos imposuit...

C'est de cette évocation des vents maintenus prisonniers sous des masses rocheuses que comme l'a noté Denis Fenney - se distingue Ovide qui, après avoir évoqué le même pouvoir destructeur de ces vents, ajoute que le démiurge a "posé sur eux » l'éther sans poids et étranger à toute impureté terrestre :

Haec super inposuit liquidum et grauitate carentem aethera nec quicquam terrenae faecis habentem.

Au-dessus d'eux, il posa l'éther limpide et privé de toute pesanteur, pur de toute la lie terrestre. ${ }^{19}$

A la reprise de la formule virgilienne insuper ... imposuit, devenue dans le texte ovidien, super imposuit, s'ajoute la parenté des deux démonstratifs hoc et haec. Cette reprise permet à Ovide de se démarquer fortement du modèle de l'Énéide, puisqu'il remplace, au-dessus des vents, la masse des montagnes par l'éther plus léger encore que ces vents et qualifié par l'expression grauitate carens, en écho à la formule sine pondere (Met. 1.26); en outre, la précision selon laquelle cet éther sans poids n'a rien de «terrestre » peut également viser à

\footnotetext{
${ }^{18}$ Verg., Aen. 1.52-63. On notera que les deux passages concernant les vents et leur force de désordre potentiel sont situés au même endroit dans les deux épopées.

${ }^{19}$ Ov., Met. 1.67-8 : «Au-dessus d'eux, il posa l'éther limpide et privé de toute pesanteur, pur de toute la lie terrestre »
} 
souligner la divergence du cosmos ovidien avec celui de l'Énéide, où les vents sont écrasés par des montagnes ${ }^{20}$.

Pour résumer, tandis que chez Virgile, les vents, vus comme une force potentielle de désordre, sont réprimés par le poids de lourdes montagnes, chez Ovide, ils sont placés sous un élément plus léger encore qu'eux, dans un passage qui se donne expressément comme une correction du modèle virgilien. Nous allons donc considérer les enjeux - idéologiques et poétiques - de cette absence de poids sur des vents laissés en liberté chez Ovide, par opposition à l'univers virgilien, où ces forces de désordre sont craintes (comme l'indique le participe metuens) et, dès lors, contenues et contrôlées. Pour cela, il nous faut commencer par rappeler la double valeur, politique et poétique, de cette question du contrôle des vents dans l'œuvre de Virgile.

\section{Sens idéologique et poétique du désordre causé par les vents dans l'Énéide.}

\section{Une métaphore politique}

Pour n'évoquer que les aspects les plus saillants de la dimension politique qui est manifestement associée à l'image du contrôle des vents chez Virgile, on rappellera tout d'abord qu'Éole est désigné comme un roi (rex) qui contient sous son pouvoir les vents

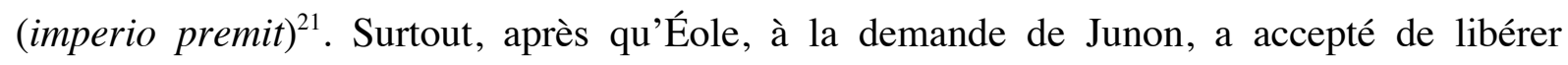
provisoirement les vents maintenus sous son empire, le tableau de la tempête qui s'ensuit donne lieu à une comparaison politique explicite, au moment où Neptune calme les éléments :

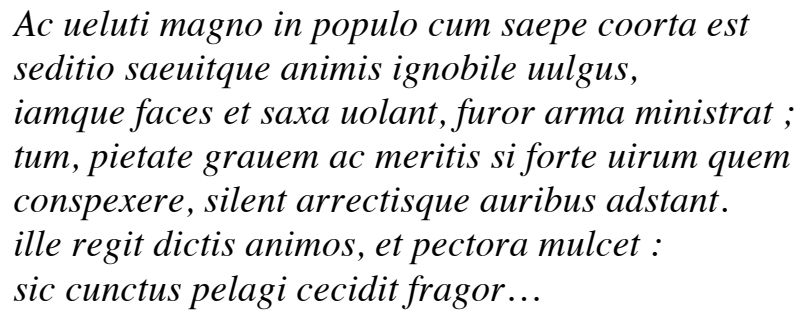

Ainsi, lorsque souvent dans un grand peuple la sédition s'est levée et que l'ignoble populace est emportée par des élans de rage, que déjà volent les torches et les pierres, et que la folie furieuse fournit les armes, alors pourvu qu'apparaisse un homme de poids, vénérable par sa piété et ses bienfaits, la foule fait silence et se tient debout, l'oreille aux aguets. Lui, par ses paroles, gouverne leurs élans et adoucit les cœurs. De même, tout le fracas de la mer retomba... ${ }^{22}$

\footnotetext{
${ }^{20}$ Le lien est plus étroit encore entre ces deux passages si l'on y considère également leurs références respectives à la description des vents que l'on trouve dans le De Rerum Natura. Voir Wheeler 1995a qui montre comment, dans ces deux vers (Met. 1.67-68), Ovide rectifie précisément la correction naguère apportée par Virgile à Lucrèce.

${ }^{21}$ Verg., Aen. 1.26-8 (texte cité supra).

${ }^{22}$ Verg., Aen. 1.148-54.
} 
les vents de tempête sont à l'image d'un peuple rebelle calmé par un homme providentiel qualifié de uir pietate grauis ac meritis -, capable d'apaiser les troubles politiques grâce au poids de l'autorité que lui confèrent sa valeur morale et ses exploits, à l'instar d'Octave, auteur et garant de l'ordre retrouvé après le désordre des guerres civiles.

En outre, le sens politique de cette image des vents de tempête est corroboré par un autre passage: le Conseil des Dieux au livre 10 de l'Énéide, qui doit être lu en regard de la description de la tempête qui ouvre l'œuvre puisque le lien métaphorique entre vents et désordre politique y est inversé. Junon (encore elle!) y sème le trouble dans l'assistance divine, suscitant des frémissement comparés à ceux des vents qui se lèvent et annoncent la tempête - avant que cette tempête (politique), précisément, soit calmée par Jupiter :

Talibus orabat Iuno ; cunctique fremebant caelicolae assensu uario : ceu flamina prima, cum deprensa fremunt siluis et caeca uolutant murmura, uenturos nautis prodentia uentos. Tum pater omnipotens, rerum cui prima potestas, infit ; eo dicente, deum domus alta silescit et tremefacta solo tellus. Silet arduus aether.

Tum Zephyri posuere, premit placida aequora pontus.

Ainsi avait parlé Junon ; et tous les habitants du ciel frémissaient de sentiments divers, tels les premiers souffles d'air qui frémissent, encore emprisonnés dans les forêts, et roulent d'invisibles murmures annonçant l'arrivée des vents pour les marins. Alors, le père tout-puissant, qui a sur le monde un pouvoir souverain, commence : tandis qu'il parle, la haute demeure des dieux est silencieuse et, en bas, la terre tremble ; l'éther élevé fait silence. Alors les Zéphyrs se sont posés, et la mer contient ses flots apaisés. ${ }^{23}$

La question du contrôle des vents possède donc, indéniablement, un enjeu idéologique, explicitement souligné par le recours aux images de la tempête cosmique et politique, réciproquement employées comme métaphore l'une de l'autre, aux livres 1 et 10 de l'Énéide.

\section{La hantise de la dispersion poétique}

En parallèle, les motifs du vent en liberté et/ou de la tempête sont également utilisés par Virgile pour thématiser, dans son œuvre, sa hantise du désordre narratif, de l'éparpillement poétique qui menacerait l'unité du poème épique. La critique a ainsi pu noter que la tempête du livre 1, causée par la libération des vents d’Éole, parce qu'elle a dévié Énée hors de sa course en le déportant sur les rives de la Libye, est à l'origine de l'excursus carthaginois et donc d'une menace pour l'unité du poème impliquant la linéarité téléologique d'une parole

\footnotetext{
${ }^{23}$ Verg., Aen. 10.96-103.
} 
épique chargée de chanter les fata qui ont amené Énée à fonder Rome ${ }^{24}$. La validité d'une telle analyse de la tempête initiale, comme la dramatisation d'une tentation digressive qui compromettrait la cohérence et l'intégrité du poème, peut être confirmée dans le texte même, semble-t-il, par une image horrifique : la vision du naufrage qui a suivi la tempête initiale, avec les débris de navires brisés parmi lesquels flottent, épars, dispersés par la tempête, les armes des héros, arma uirum ${ }^{25}$, autrement dit, une métaphore de l'épopée virgilienne ellemême (ainsi désignée par son incipit, dont la valeur réflexive peut être soulignée par les tabulae, des planches de bois qui surnagent dans le naufrage mais aussi - à un autre niveau les tablettes à écrire) qui semble menacée, dans son intégrité, par la tempête initiale ${ }^{26}$. Virgile semble nous livrer là la vision cauchemardesque d'une œuvre éparpillée, détruite par les vents de tempête. Mais cette hantise de l'éparpillement poétique est conjurée puisque ces mêmes vents sont contenus par le poids des montagnes dans la cave d’Éole, et que, même libérés à dessein par ce dernier, ils sont maîtrisés par « un homme de poids » (uir grauis), qui est non seulement l'image du bon chef d'état, mais aussi, peut-être l'image du poète ${ }^{27}$.

Outre cette vision fantasmée, une seconde représentation du vent comme source de désordre poétique - envisagé négativement, comme un risque heureusement évité - laisse entrevoir ailleurs cette même hantise de la dispersion de l'œuvre : il s'agit de la description que fait Hélénus de la cave de la Sibylle. Cette dernière y chante les destins (fata canit), tout comme le poète épique ; d'ailleurs, elle transcrit des chants (carmina) sur des feuilles qu'elle dispose en ordre immobile jusqu'à ce qu'y pénètre le vent qui en perturbe l'ordre :

Fata canit, foliisque notas et nomina mandat.

Quaecumque in foliis descripsit carmina uirgo, digerit in numerum, atque antro seclusa relinquit.

Illa manent immota locis, neque ab ordine cedunt.

Verum eadem, uerso tenuis cum cardine uentus

impulit et teneras turbauit ianua frondes,

numquam deinde cauo uolitentia prendere saxo

nec reuocare situs aut iungere carmina curat.

$<$ la Sibylle qui> chante les destins et confie à des feuilles les signes et les noms. Toutes les prophéties que la vierge a écrites sur ces feuilles, elle les classe et les dispose, puis les

\footnotetext{
${ }^{24}$ Voir notamment Deremetz 1994. On trouve par ailleurs dans les Métamorphoses la reconnaissance auctoriale de cette image virgilienne de la tempête comme tentation digressive refusée, et qu'Ovide pour sa part (et contre Virgile) développe pleinement, suivant en cela le refus callimachéen d'un poème un et continu : voir Klein 2011. ${ }^{25}$ Verg., Aen. 1.118-9 : Apparent rari nantes in gurgite uasto / arma uirum tabulaeque et Troia gaza per undas, çà et là apparaissent de rares naufragés nageant dans le vaste gouffre, les armes des héros, des planches et les richesses de Troie emportées par les ondes.

${ }^{26}$ Oliensis 2004, 31 .

${ }^{27}$ Pour une analyse de la formule regit dictis en ce sens (et la suggestion du lien inextricable entre poétique et politique ici) : Nelis 2011, 281-2.
} 
laisse à l'intérieur de son antre. Celles-ci demeurent immobiles où elles sont, et restent bien en ordre. Mais lorsque, la porte tournant sur ses gonds, un mince souffle de vent a bousculé et a mélangé, dans un courant d'air, les tendres frondaisons, jamais ensuite elle ne se soucie de rattraper les prophéties qui voltigent dans sa caverne, ni de les remettre à leur place et de les joindre entre elles. ${ }^{28}$

Dans cette évocation, on retrouve le risque conjuré du possible désordre qui pourrait menacer le chant des fata et qui est, cette fois encore, associé à l'image du vent. C'est pourquoi au livre 6, fort de cet avertissement, Énée demandera à la Sibylle de ne pas confier ses carmina à ces feuilles fragiles, jouets des vents qui peuvent les emporter à leur gré :

... Foliis tantum ne carmina manda, ne turbata uolent, rapidis ludibria uentis.

Seulement, ne confie pas tes prophéties à des feuilles, afin qu'elles ne s'envolent pas en désordre, jouets des vents rapides. ${ }^{29}$

On voit donc que l'ordre (ordo) dont le chant des fata ne doit pas s'écarter, l'intégrité et l'unité linéaire de l'œuvre poétique sont un enjeu thématisé, au sein de l'Énéide même, par ces images de vents de tempête qui disent la hantise d'un désordre narratif, craint mais conjuré et maîtrisé.

Avec la question du contrôle (ou non) des vents comme source de désordre - que ce désordre soit politique ou poétique -, c'est donc sur cette problématique inhérente à toute épopée et en particulier à l'Énéide que les Métamorphoses se positionnent par rapport à cette dernière, en faisant d'elle, d'une certaine manière, un modèle autant qu'un contre-modèle.

Il s'agit alors de voir comment ces images de désordre potentiel, évoquées comme des tentations finalement refusées et remises 'sous contrôle' dans l'Énéide, ont suscité chez Ovide la création dans son œuvre de deux 'avatars' virgiliens : d'une part, la radicalisation d'un contre-modèle exacerbé, en la personne de Jupiter, dont nous verrons qu'il se comporte (temporairement, du moins) comme un dieu épique, plus 'virgilien' encore qu'il ne l'était chez Virgile même, et qui est en cela déplacé, illégitime, dans l'univers épique ovidien; d'autre part, le développement de ces hantises conjurées, grâce à la figure de Fama (ce qui n'est certainement pas fortuit dans la mesure où cette déesse montrueuse incarnait déjà, chez Virgile, la tentation d'une épopée autre ${ }^{30}$ ).

\footnotetext{
${ }^{28}$ Verg., Aen. 3.444-451.

${ }^{29}$ Verg., Aen. 6.74-75.

${ }^{30}$ Voir Clement-Tarantino 2006.
} 


\section{Deux 'avatars' virgiliens dans les Métamorphoses : Jupiter et Fama.}

\section{Jupiter et le Conseil des dieux (Met. 1) ou Virgile radicalisé.}

Avec la figure de Jupiter, au début de l'œuvre, Ovide semble intégrer dans son propre texte 'son' contre-modèle virgilien tout en radicalisant l'altérité que ce dernier représente par rapport à son propre projet épique. Cela passe par un jeu intertextuel précis dans lequel Ovide superpose les deux hypotextes virgiliens où interviennent les sens propre et figuré des vents de la tempête et du soulèvement politique, respectivement employés comme métaphore l'un(e) pour l'autre : en effet, le Conseil des dieux présenté au début des Métamorphoses d'une part décalque celui d'Énéide 10, en ce qu'il évolue, lui aussi, en tempête métaphorique apaisée - ou plutot, en l'occurrence, réprimée - par Jupiter, tout en étant placé, d'autre part, au livre 1 , à l'endroit du poème où Virgile avait, quant à lui, situé la tempête réellement déchaînée par la libération des vents d'Éole sur ordre de Junon.

Dans le passage ovidien, donc, le Conseil des dieux est réuni par Jupiter suite à une tentative d'attentat dont il a été victime et dont il fait le récit à son auditoire : le grand mouvement de trouble que cela suscite dans l'assemblée divine est alors aussitôt réprimé par le roi des dieux lui-même :

\footnotetext{
Confremuere omnes studiisque ardentibus ausum talia deposcunt. Sic, cum manus impia saeuit sanguine Caesaro Romanum extinguere nomen, attonitum tanto subitae terrore ruinae humanum genus est totusque perhorruit orbis. Nec tibi grata minus pietas, Auguste, tuorum est quam fuit illa Ioui. Qui postquam uoce manuque murmura compressit, tenuere silentia cuncti. Substitit ut clamor pressus grauitate regentis, Iuppiter hoc iterum sermone silentia rupit.
}

Tous frémirent et, brûlant de zèle, demandent que soit châtiée une telle audace. Ainsi, lorsqu'une troupe impie s'acharna, dans sa rage, à éteindre le nom de Rome dans le sang de César, le genre humain fut frappé d'un immense effroi devant ce désastre soudain et l'univers entier en frissonna d'horreur. Et ce témoignage de l'attachement des tiens ne te fut pas moins doux, Auguste, que celui-ci ne le fut à Jupiter. Après que ce dernier, de la voix et du geste, eut réprimé les murmures, tous gardèrent le silence. Lorsque les cris se furent arrêtés, sous le poids de sa royale gravité, Jupiter, de nouveau, rompit le silence par ces paroles. ${ }^{31}$

Les dieux frémissent tous (Met. 199, confremuere omnes) en réaction aux paroles de Jupiter comme ils l'avaient fait, dans le texte de Virgile, en réaction à celles de Junon (Aen. 10.96, cuncti ...fremebant), ce en quoi ils y étaient explicitement comparés aux souffles frémissants

\footnotetext{
${ }^{31}$ Ov., Met. 1.199-208.
} 
(flamina ...fremunt) dont les murmures (murmura) annoncent aux marins les tempêtes ${ }^{32}$, et donc implicitements assimilés aux vents libérés par l'entremise de cette même Junon lors de la tempête initiale (cf. Aen. 1.55, magno cum murmure ...fremunt, pour les vents dans la cave d'Éole). Or ce sont ces mêmes «murmures» des vents que réprime le Jupiter des Métamorphoses, dans un second temps (Met.1.206, murmura compressit). Il en résulte le silence général: tenuere silentia cuncti (ibid.): la reprise du terme cuncti tisse un lien supplémentaire avec le texte d'Énéide 10 (cf. cuncti fremebant) tandis que le silence qu'obtient le Jupiter ovidien est comparable à celui qu'avait obtenu le Jupiter virgilien dans le Conseil des dieux de l'Énéide (silescit ; silet) ${ }^{33}$, mais également «l'homme de poids » (uir... grauis) capable de calmer la sédition populaire (Aen. I, 152, silent) auquel était comparé Neptune apaisant les vents d'Éole au livre 1 de l'Énéide.

C'est d'ailleurs précisément à cette dernière comparaison que fait écho l'interpellation directe d'Auguste, expréssement comparé à Jupiter lors du Conseil des dieux des Métamorphoses : par ce parallèle explicite entre l'histoire mythologique et la situation politique contemporaine, Ovide s'amuse avec le lecteur de l'Énéide qui avait reconnu la figure d'Octave / Auguste dans le uir pietate ac meritis grauis auquel Virgile avait comparé Neptune calmant les vents de tempête ${ }^{34}$. Il est surtout intéressant alors de constater que la valeur de grauitas, attribuée par Virgile à l'homme d'État auquel sa vertu et ses mérites confèrent le poids d'une autorité légitime, devient chez Ovide la grauitas regentis qu'exerce Jupiter pour réprimer l'agitation bruyante de la foule: Met.1.207, clamor pressus grauitate regentis. Le verbe premere redouble d'ailleurs son composé compremere employé au vers précédent (Met.1.206, murmura compressit) pour dire la répression (/ l'écrasement) par Jupiter des «murmures » de son public, qui faisaient écho à ceux des vents en Énéide 1 et à ceux de l'assistance divine en Énéide 10 respectivement dominés par Éole et apaisés par Jupiter. Or cette répétition de (com)premere tend indéniablement à actualiser le sens concret du poids associé à la grauitas comme supposée valeur morale du dirigeant politique, vue ici comme un poids par lequel on réprime les soulèvements de la foule. Il n'est alors pas anodin de noter que ce verbe premere

\footnotetext{
${ }^{32}$ Verg., Aen. 10.96-99 : cunctique fremebant / caelicolae assensu uario : ceu flamina prima, / cum deprensa fremunt siluis et caeca uolutant / murmura, uenturos nautis prodentia uentos, « et tous les habitants du ciel frémissaient de sentiments divers, tels les premiers souffles d'air qui frémissent, encore emprisonnés dans les forêts, et roulent d'invisibles murmures annonçant l'arrivée des vents pour les marins. »

${ }^{33}$ Ibid. 100-103 : Tum pater omnipotens, rerum cui prima potestas, / infit ; eo dicente, deum domus alta silescit / et tremefacta solo tellus. Silet arduus aether. / Tum Zephyri posuere, premit placida aequora pontus : « Alors, le père tout-puissant, qui a sur le monde un pouvoir souverain, commence : tandis qu'il parle, la haute demeure des dieux est silencieuse et, en bas, la terre tremble ; l'éther élevé fait silence. Alors les Zéphyrs se sont posés, et la mer contient ses flots apaisés ».

${ }^{34}$ Barchiesi 2005, 187. Voir aussi, pour l'interprétation de cette explicitation de la comparaison virgilienne, Rosati 2001, 45-46.
} 
est utilisé, quant à lui, dans la description de la cave d'Éole maintenant les vents sous son pouvoir (Aen . 1.54, imperio premit) et sous le poids d'imposantes montagnes...

Ainsi, l'équivalence structurelle entre les deux passages, respectivement situés au livre 1 de l'Énéide ou des Métamorphoses, dans lesquels le pouvoir divin comme garantie de l'ordre est (plus ou moins ouvertement) identifié à celui du princeps, permet au texte ovidien de gloser son hypotexte virgilien. La grauitas comme qualité suprême de l'homme d'Etat virgilien (le uir pietate grauis ac meritis), qui le rend capable de faire cesser les désordres populaires, est réinterprétée par Ovide comme le poids d'un pouvoir répressif, écrasant des troubles qu'il a par ailleurs lui-même suscités ! Cette glose se donne alors comme une explicitation des sousentendus virgiliens sur lesquels se fonderait la légitimation de l'autorité divine: les rapprochements latents opérés dans l'Énéide sont mis au jour par Ovide qui révèle la véritable nature de la grauitas assimilée au pouvoir politique. Ainsi, avec l'image des murmura de l'auditoire divin - qui sont apparentés, par le biais des références superposées au livre 10 et au livre 1 de l'Énéide, aux murmures frémissants des vents enfermés dans la cave d'Éole - ici écrasés par le roi des dieux (murmura compressit), Ovide souligne le lien implicite entre les deux images virgiliennes, que sont, d'une part, la grauitas de l'homme d'état auquel est comparé Neptune apaisant les vents d'Éole, et, de l'autre, le moyen par lequel Jupiter avait d'abord maîtrisé et contenu la force destructrice de ces mêmes vents : les emprisonner sous le pouvoir d'un roi (rex Aeolus... imperio premit), et concrètement, les enfermer sous la masse pesante de hautes montagnes.

Avec la figure de Jupiter pesant du poids de sa « royale gravité » sur ses sujets pour réprimer leur manifestation de zèle, l'auteur des Métamorphoses semble donc intégrer dans son poème une représentation du fonctionnement épique virgilien, réinterprété et radicalisé pour y figurer le contre-modèle de l'épopée ovidienne, et ce grâce à un détail qui pourrait apparaître fortuit mais qui est bien plus significatif qu'il n'y paraît dans la mesure où, dans ce jeu de reprises serrées, tout fait système.

De fait, avant que s'ouvre le Conseil des dieux, Ovide a pris de soin de préciser la situation de Jupiter dans le ciel (et, donc, le lieu d'où il ne tardera pas à exercer le poids de son autorité royale (grauitas regentis) comparé par le jeu de l'intertextualité au poids des lourdes montagnes pesant sur les vents dans la cave d'Éole) :

Quae pater ut summa uidit Saturnius arce... 
Lorsque, du haut des hauteurs suprêmes, le père des dieux, né de Saturne, vit cela... ${ }^{35}$

La demeure du roi des dieux est située summa ... arce, dans les hauteurs suprêmes, autrement dit aux lieux où lors de la cosmogonie initiale s'était élevé, du fait de sa légèreté, l'éther « sans poids »,

\section{Ignea conuexi uis et sine pondere caeli emicuit summaque locum sibi fecit in arce}

L'essence ignée et sans poids de la voûte céleste, s'élança et s'installa dans les hauteurs suprêmes... ${ }^{36},-$

cette légèreté annonçant la 'correction' ovidienne du cosmos virgilien quelques vers plus loin, puisque cet éther, placé au sommet du monde, y était alors dit «affranchi de toute pesanteur » (grauitate carentem), lorsque le démiurge avait choisi de le poser sur les vents impétueux ${ }^{37}$, contrairement au Jupiter de l'Énéide qui avait placé sur ces mêmes vents le poids de lourdes montagnes.

Autrement dit - et pour le formuler avec quelque provocation - le Jupiter ovidien se comporte comme s'il n'avait pas perçu cette correction ovidienne du cosmos épique virgilien, en imposant (sur le modèle d'Éole au seuil de l'Énéide), le poids de son autorité (grauitas) du haut de ce lieu où ne devrait régner qu'un élément plus léger encore que les vents laissés en liberté $^{38}$. Ce faisant Ovide, qui représente par cette figure de dirigeant divin le fonctionnement de l'épopée virgilienne (non sans l'avoir par là-même réinterprété et radicalisé) le désigne en même temps comme un contre-modèle hétérogène et 'déplacé' - au sens propre du terme dans le système épique qui est le $\operatorname{sien}^{39}$.

\section{La demeure de Fama}

Ce statut initial de Jupiter, que l'on pourrait qualifier de dieu virgilien égaré dans l'univers épique ovidien, est à lire en regard avec celui d'une autre figure qui se présente comme une alternative : Fama, la Rumeur. De fait, sur le plan cosmologique on constate que Jupiter et

\footnotetext{
${ }^{35}$ Ov., Met. 1.163.

${ }^{36}$ Ov., Met. 1.26-27.

${ }^{37}$ Ov., Met. 1.67-8 : Haec super inposuit liquidum et grauitate carentem /aethera nec quicquam terrenae faecis habentem, «Au-dessus d'eux, il posa l'éther limpide et privé de toute pesanteur, pur de toute la lie terrestre ».

${ }^{38}$ On voit alors qu'il était indispensable, pour Ovide, de bien distinguer le démiurge, créateur du cosmos, et Jupiter (contrairement à l'Enéide où c'est le roi des dieux qui impose aux vents de lourdes montagnes pour en contrôler les forces de désordre), dans la mesure où ce dernier 'ne respecte pas' l'agencement de l'univers ovidien tel que le démiurge l'a mis en place.

${ }^{39}$ En plus d'être 'cosmologiquement déplacée' dans l'univers épique des Métamorphoses, la grauitas regentis de Jupiter y est aussi provisoire puisque le dieu ne tardera pas à s'en débarrasser, constatant qu'elle est incompatible avec l'amour qu'il éprouve pour Europe (et avec sa propre métamorphose en taureau pour séduire cette dernière) : cf. sceptri grauitate relicta (Ov., Met. 2.847).
} 
Fama ont tous deux établi leur résidence dans les régions supérieures de l'univers d'où ils observent le monde : c'est ainsi qu'à la situation surplombante du palais de Jupiter,

Quae pater ut summa uidit Saturnius arce... ${ }^{40}$

s'apparente celle de la maison de Fama, également située dans les hauteurs les plus élevées du cosmos :

Fama tenet summaque domum sibi legit in arce

$[\ldots]$

Ipsa, quid in caelo rerum pelagoque geratur

et tellure, uidet totumque inquirit in orbem.

Là réside la Renommée. Elle a choisi sa demeure dans les hauteurs suprêmes.

[...]

Elle-même, tout ce qui se passe dans ciel, dans la mer et sur la terre, elle le voit et elle surveille l'univers entier. ${ }^{41}$

Ces deux maisons sont donc situées au même lieu du cosmos (summa arx), d'où elles permettent de contrôler, chacune à sa manière, l'ensemble de l'univers ${ }^{42}$. Surtout, ce rapprochement par la formule summa arx me semble intéressante par rapport à l'organisation cosmique des Métamorphoses telle qu'elle a été mise en place par le démiurge.

Comme nous l'avons vu, la situation de Jupiter et de sa puissance pesante dans cette région supérieure est en contradiction avec l'organisation du cosmos ovidien selon lequel cette summa arx est un lieu réservé à la légèreté de l'éther qui surplombe les vents sans peser sur eux, sans exercer de contrôle sur les désordres qu'ils peuvent potentiellement provoquer. On pourrait alors faire l'hypothèse que la maison de Fama nous offre, en cela, une alternative au contrôle de l'univers par une grauitas regentis cosmologiquement 'déplacée', et qui serait, elle, au contraire, conforme à la cosmogonie ovidienne selon laquelle les vents turbulents ne sont soumis à aucun contrôle.

Et, de fait, cette hypothèse peut être vérifiée, ou du moins fortement corroborée, grâce aux jeux de l'intertextualité, qui relient l'évocation de cette maison de Fama et celles des deux tempêtes, réelle et métaphorique, de l'Énéide 1 et 10 , qui avaient déjà été reprises - et réinterprétées - au livre 1 des Métamorphoses : c'est donc au sein du réseau intertextuel tissé entre ces quatre passages que prennent sens ces reprises serrées de termes récurrents, si anodins puissent-ils sembler séparément et isolément ${ }^{43}$. Examinons donc un extrait de

\footnotetext{
${ }^{40}$ Ov., Met. 1.163 : Lorsque, du haut des hauteurs suprêmes, le père des dieux, né de Saturne, vit cela...

${ }^{41}$ Ov., Met. $12.43 ; 62-63$.

${ }^{42}$ Cf. uidit (1.163) et uidet (12.63).

${ }^{43}$ Sur les correspondances structurelles qui unissent la maison de Fama dans les Métamorphoses et la tempête d'Énéide 1, voir Hardie 2002a, 76.
} 
l'ekphrasis de la maison de Fama. Tout n'y est que frémissement (fremit) et murmures (murmura), sans silence (silentia) en aucun lieu :

Tota fremit uocesque refert iteratque quod audit.

Nulla quies intus nullaque silentia parte ;

nec tamen est clamor, sed paruae murmura uocis.

Tout en elle est frémissement, elle rapporte des voix et répète ce qu'elle entend. A l'intérieur, point de calme nulle part, point de silence. Ce ne sont pas des cris, pourtant, mais des murmures chuchotés d'une petite voix ${ }^{44}$.

Les frémissements et les murmures qui se donnent libre cours dans la maison de Fama semblent alors devoir être lus comme l'équivalent des vents qu'apaiseront les dieux virgiliens (Neptune puis Jupiter respectivement en Énéide 1 et 10) et qui seront - plus explicitement réprimés, voire écrasés (pressit; compressit) par le Jupiter ovidien en Métamorphoses $1^{45}$. Dès lors, l'apparente absence de contrôle que Fama exerce sur eux ${ }^{46}$ semble faire d'elle l'alternative ovidienne au Jupiter 'virgilien (celui de l'Énéide, ou l'avatar de dieu virgilien qu'Ovide a intégré dans sa propre épopée tout en le désignant comme hétérogène et illégitime), et ce, en toute conformité, cette fois-ci, avec l'organisation cosmique par laquelle l'auteur des Métamorphoses s'était fortement distingé de son prédécesseur.

Or cette liberté des vents métaphoriques que Fama ne réprime pas, sur lesquelles elle ne pèse pas, a également une double valeur, idéologique et poétique. Commençons par dire quelques mots de son sens politique. Tout d'abord, la description de la demeure de Fama semble convoquer précisément comme modèle, à l'intérieur de la tempête du livre 1 de l'Énéide, la comparaison virgilienne des vents calmés par Neptune avec les manifestations violentes d'une révolte populaire apaisée par la vision du uir grauis :

Ac ueluti magno in populo cum saepe coorta est seditio saeuitque animis ignobile uulgus,

\footnotetext{
${ }^{44}$ Ov., Met. $12.47-49$

${ }^{45}$ Le passage reprend les termes virgiliens auxquels faisait déjà écho le Conseil des dieux au livre 1, mais en les inversant. Cf. tota fremit, ici, et fremunt (pour les vents dans la cave d'Eole), cuncti fremebant (pour la tempête » politique » soulevée par Junon en Aen. 10) ; confremuere omnes (pour le Conseil des dieux de Met. 1) mais chez Fama, ces frémissements apparentés à ceux des vents ne sont pas apaisés / réprimés. De même, les paruae murmura uocis rappellent les «murmures » des vents réels ou métaphoriques que l'on rencontre dans ces trois passages : cf magno cum murmure (Aen. 1); uolutant murmura (Aen. 10) ; murmura compressit (Met. 1), mais là encore ceux que l'on entend dans la maison de Fama ne sont pas réprimés. Enfin, la précision nulla silentia parte semble s'opposer précisément au silence qui règne après que les souffles des vents ont été calmés / écrasés dans les autres passages: Aen. 1.152: silent; Aen. 10.101: domus alta silescit; 102 : silet arduus aether; Met. 1.206 : tenuere silentia cuncti.

${ }^{46}$ Hardie 2002a, 76-77.
} 
Ainsi, lorsque souvent dans un grand peuple la sédition s'est levée et que l'ignoble populace est emportée par des élans de rage... ${ }^{47}$

On note en effet la présence parmi les compagnes de Fama de Seditio (Met. 12.61 : Seditio... recens, «la Sédition soudaine »), tandis que l'image du peuple est reprise - l'ignobile uulgus qui se soulève avant de céder devant l'autorité naturelle et légitime du dirigeant idéal chez Virgile devenant chez Ovide le leue uulgus qui fréquente la maison de Fama:

Atria turba tenet : ueniunt, leue uulgus, euntque...

La foule emplit l'atrium, ils vont et viennent, peuple léger... ${ }^{48}$

En outre, on peut remarquer que ce terme ignobilis trouve son contraire avec l'évocation de la noblesse divine (deorum nobilium) qui se presse dans les atriums, non de la demeure de Fama, mais du palais de Jupiter :

Hac iter est superis ad magni tecta Tonantis regalemque domum. Dextra laeuaque deorum atria nobilium ualuis celebrantur apertis.

C'est par ce chemin que les dieux d'en haut vont chez le grand Jupiter Tonnant, dans sa demeure royale. A droite et à gauche, les atriums aux portes ouvertes sont fréquentés par les dieux nobles à qui ils sont réservés. ${ }^{49}$

Avec cette mention des atria des maisons de Fama et de Jupiter respectivement fréquentés pour l'une par la foule (turba) et l'équivalent de l'ignobile uulgus virgilien, ici qualifié de leue uulgus, et pour l'autre par les dieux nobles (nobilium), il semble là encore que ces deux passages peuvent être rapprochés - à la fois en eux-mêmes et par leur commune référence à un texte précis de l'Énéide -, et ce, pour être mieux opposés l'un à l'autre... ${ }^{50}$.

Il en va de même si l'on considère le non-contrôle de Fama sur le «peuple léger » qu'elle abrite dans sa demeure en y lisant un sens métapoétique, ce qu'il serait difficile de ne pas faire ici dans la mesure où ce leue uulgus est en réalité un peuple de paroles, fait de mensonges joints à la vérité (à l'instar de ceux des Muses hésiodiques) qui errent en tous sens, de mots qui roulent pêle-mêle ${ }^{51}$ :

\footnotetext{
${ }^{47}$ Verg., Aen. 1.148-149.

${ }^{48}$ Ov., Met. 12.53 .

${ }^{49}$ Ov., Met. 1.170-172.

${ }^{50} \mathrm{~J}$ 'en reste là sur ce point, et pour une lecture en termes politiques de la Fama ovidienne, je renvoie à un article de William Gladhill qui propose de voir dans la Fama ovidienne une image de la fama forensis républicaine et dans sa maison l'équivalent cosmologique du Forum Romain qui, du point de vue du «Palatin du ciel » où se trouve le palais de Jupiter, représente une force de sédition : voir Gladhill à paraître.

${ }^{51}$ Que ce « peuple » de paroles soit ici qualifié de leuis est par ailleurs signifiant, au regard de l'importance de la leuitas comme catégorie poétique choisie, revalorisée et mise en avant par Ovide pour caractériser son écriture poétique : voir Klein 2008.
} 
Atria turba tenet : ueniunt, leue uulgus, euntque

mixta cum ueris passim commenta uagantur

milia rumorum confusaque uerba uolutant.

La foule emplit l'atrium, ils vont et viennent, peuple léger, mille bruits inventés, mêlés aux vrais, errent en tous sens et roulent des paroles confuses ${ }^{52}$.

On peut alors postuler que ces sons, ces paroles fictives et ces mots qui errent en désordre dans la maison de Fama représentent une certaine vision de l'écriture épique, qu'Ovide propose comme alternative à un contre-modèle virgilien radicalisé, tout en revendiquant, pour sa part, les tentations esquissées puis refusées de l'Énéide.

On pourrait ainsi signaler que l'évocation des mots poétiques qui vont et viennent librement dans la maison de Fama rappelle celle des vents de la tempête métaphorique soulevée par Junon en Énéide 10 avant d'être maîtrisée par Jupiter.

... Ceu flamina prima,

Cum deprensa fremunt siluis et caeca uolutant

Murmura, uenturos nautis prodentia uentos.

... tels les premiers souffles d'air qui frémissent, encore emprisonnés dans les forêts, et roulent d'invisibles murmures annonçant l'arrivée des vents pour les marins. ${ }^{53}$

Dans le contexte de la reprise de termes-clés (cf. fremunt - tota fremit ; murmura - paruae murmura uocis, etc...), la répétition de la forme uolutant dans les deux cas située en fin de vers nous incite à comparer les mots qui roulent pêle-mêle, dans le texte ovidien, avec les souffles qui annoncent les vents de tempêtes dans l'hypotexte virgilien. En outre, ces paroles qui tournoient en se mélangeant (confusa uerba uolutant) font également penser aux carmina uolitentia, les chants des destins dé-rangés par le vent, qui voltigent et s'envolent en tous sens dans la grotte de la Sibylle ${ }^{54}$. Autrement dit, dans la demeure de la Fama ovidienne se réalise non seulement l'équivalent de la tempête politique maîtrisée par Éole, Neptune et Jupiter dans l'Énéide, mais aussi de la tempête poétique, du désordre narratif : la dispersion des mots

\footnotetext{
${ }^{52}$ Ov., Met. 12.53-55 : «La foule emplit l'atrium, ils vont et viennent, peuple léger, mille bruits inventées, mêlés aux vrais, errent en tous sens et roulent des paroles confuses. »

${ }^{53}$ Verg., Aen. 10.97-99.

${ }^{54}$ Verg., Aen. 3.448-451: Verum eadem, uerso tenuis cum cardine uentus / impulit et teneras turbauit ianua frondes, / numquam deinde cauo uolitentia prendere saxo /nec reuocare situs aut iungere carmina curat. « Mais lorsque, la porte tournant sur ses gonds, un mince souffle de vent a bousculé et a mélangé, dans un courant d'air, les tendres frondaisons, jamais ensuite elle ne se soucie de rattraper les prophéties qui voltigent dans sa caverne, ni de les remettre à leur place et de les joindre entre elles ». Peut-être pourrait-on déceler, en outre, dans l'allusion ovidienne à ce passage un jeu de mot si dans l'image de la foule (turba) des mots qui occupent la maison de Fama on entend un écho du verbe turbare, l'évocation ds poèmes potentiellement mélangés, mis en désordre dans la cave de la Sibylle. Cf. aussi Verg., Aen. 6.74-75 : Foliis tantum ne carmina manda, / ne turbata uolent, rapidis ludibria uentis, "Seulement, ne confie pas tes prophéties à des feuilles, afin qu'elles ne s'envolent pas en désordre, jouets des vents rapides ».
} 
emportés à tout vent, crainte (et empêchée) par Hélénus et Énée, et derrière eux, bien sûr Virgile.

De plus, cette hantise, thématisée et conjurée dans le poème virgilien, de l'éparpillement des fata, écrits sur des feuilles trop mobiles que les vents mettent en désordre, trouve par ailleurs une réponse nette chez Ovide, au livre 15 des Métamorphoses, où Jupiter répète (à une Vénus inquiète, et mauvaise lectrice de Virgile !) le discours qu'il lui avait 'déjà' tenu au livre 1 de l'Énéide ${ }^{55}$. Or pour décrire cet hypotexte, le Jupiter ovidien évoque les destins gravés de manière impérissable dans l'acier (incisa adamante perenni / fata $^{56}$ ), qu'il a lus dans un ouvrage de bronze et de fer massif, qui - d'après le résumé qu'il en fait ensuite - s'apparente au poème virgilien lui-même, qu'aucun vent donc ne risque d'ébranler... ! ${ }^{57}$. Telle est la vision de l'Énéide que nous livre avec humour Ovide par la bouche de son Jupiter, représentant d'un Virgile érigé en contre-modèle à l'altérité radicalisée.

Pour résumer, tout se passe donc comme si Ovide affirmait : (1) mon prédécesseur craint le vent du désordre poétique dans son œuvre, mais cela ne risque guère de se produire car il a tellement conjuré cette hantise d'un poème que le vent pourrait disperser que le résultat est un poème des fata figé dans l'airain inébranlable ; (2) quant à moi, par contre, je développerai pleinement cette tentation qu'il a aussitôt refusée, cette liberté poétique des mots emportés en tous sens par des vents que ne surplombe aucun contrôle pesant ${ }^{58}$.

C'est ainsi que l'on peut lire, avec Jupiter et Fama qui partagent le même espace (summa arx) dans le cosmos des Métamorphoses (l'une de manière légitime, l'autre, d'une manière illégitime comme une instance virgilienne égarée dans l'univers épique ovidien), deux figures qui incarnent ce geste double d'Ovide à l'égard de son prédécesseur : il radicalise l'altérité

\footnotetext{
${ }^{55}$ Ov., Met. 15.807-842.

${ }^{56}$ Ibid., 813-814 : « les destins gravés dans l'acier inaltérable ».

${ }^{57}$ Sur ce passage, voir Barchiesi 2001, 129-140, et en particulier p. 131. On voit alors comment, avec cette représentation d'une Énéide gravée, solidement figée, dans l'airain, Ovide construit rétrospectivement l'image d'un Virgile radicalement 'autre' pour mieux se démarquer de lui.

${ }^{58} \mathrm{Je}$ me suis cantonnée ici, pour cela, à une lecture de la maison de Fama, en opposition à celle de Jupiter et en relation avec l'organisation du cosmos ovidien et les passages virgiliens sur la question du contrôle (ou non) des vents. Mais le développement ovidien de la tentation digressive conjurée par l'épopée virgilienne, avec l'image des mots poétiques emportés à tout vent, peut être analysé en d'autres endroits de l'œuvre, et notamment dans le proème si l'on accepte (suivant la suggestion d'Oliensis 2004, 32) de lire dans la formule fert animus (Met. 1.1)

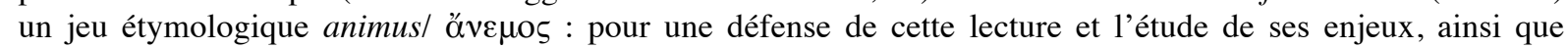
l'analyse d'autres passages corroborant l'hypothèse d'une revendication ovidienne du désordre causé par les vents en liberté comme principe poétique guidant son écriture poétique : Klein 2008,655-671.
} 
virgilienne par rapport à son propre univers épique, à la fois idéologique et poétique, et en même temps il s'adonne aux tentations refusées, aux hantises conjurées par ce dernier, pour les développer pleinement.

\section{Florence Klein,}

Université Lille 3, Halma-Ipel UMR 8164

\section{$\underline{\text { Bibliographie }}$}

Barchiesi, A. (2001), Speaking Volumes: Narrative and Intertext in Ovid and other Latin Poets, Duckworth, Londres.

Barchiesi, A. (2005), Ovidio. Metamorfosi. Volume I (Libri I-II), Milan.

Clément-Tarantino, S. (2006), Fama ou la renommée du genre. Recherches sur la représentation de la tradition dans l'Enéide, Thèse de doctorat soutenue à l'Université Lille 3 le 4 décembre 2006.

Coleman, R. (1971), «Structure and Intention in the Metamorphoses», CQ n.s. 21, 461-477.

Deremetz, A. (1994), « Fatum et Fortuna ou la métaphysique du récit virgilien », dans Thomas, J. dir., L'imaginaire religieux gréco-romain, Presses Universitaires de Perpignan, 151-166.

Deremetz, A. (1995), Le Miroir des Muses. Poétiques de la réflexivité à Rome, Lille.

Fabre-Serris, J. (1995), Mythe et poésie dans les Métamorphoses d'Ovide. Fonctions et significations de la mythologie dans la Rome augustéenne, Paris.

Farrell, J. et D. Nelis dir. (à paraître), Augustan Poetry and the Roman Republic, Oxford University Press.

Feeney, D. (1991), The Gods in epic. Poets and Critics of the Classical Tradition, Oxford University Press.

Gladhill, W. (à paraître), «The Domus of Fama and Republican Space in Ovid's Metamorphoses » dans Farell \& Nelis à paraître.

Hardie, Ph. (1993), The Epic Successors of Virgil. A study in the Dynamics of a Tradition, Cambridge. Hardie, Ph. (2002a), «'Why is Rumor Here?' Tracking Virgilian and Ovidian Fama », Ordia Prima 1, p. 67-80.

Hardie, Ph. (2002b), The Cambridge Companion to Ovid, Cambridge.

Hardie, Ph. (2009), "La 'critica contrastiva' dei poeti classici: pratica moderna e pre-moderna », SIFC s. 4, 7, 79-104.

Hinds, S. (1997), « Do-It-Yourself Literary Tradition: Statius, Martial and Others », MD 39, 187-207.

Hinds, S. (1998), Allusion and Intertext. Dynamics of appropriation in Roman poetry, Cambridge University Press, Cambridge.

Klein, F. (2008), La leuitas dans l'œeuvre ovidienne. Etude d'une catégorie poétique dans le système littéraire de la Rome augustéenne, Thèse de doctorat soutenue à l'Université Lille 3 le 29 novembre 2008.

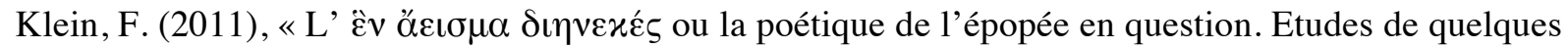
manifestations de la uox poetae dans les Métamorphoses d'Ovide », dans Raymond 2011, 335-357.

Leach, E. (1974), «Ekphrasis and the Theme of Artistic Failure in Ovid's Metamorphoses », Ramus 3, 102-142.

Maurach, G. (1979), «Ovids Kosmologie: Quellenbenutzung und Traditionsstiftung », Gymnasium 86, 131-148.

Sauron, G. (1982), « Discours symbolique et formes décoratives à Rome à l'époque augustéenne. Problèmes de méthode », MEFRA 94, 699-713.

Nelis, D. (2011), «Didactic Voices in Vergil's Aeneid», dans Raymond 2011, 275-283.

O'Hara, J. (1995-1996), «Vergil's Best Reader: Ovidian commentary on Vergilian Etymological Wordplay », CJ 91, 255-276.

Oliensis, E. (2004), « Sibylline syllables: the intratextual Aeneid», PCPS 50, 29-45. 
Otis, B. (1970), Ovid as an epic poet, Cambridge, Cambridge University Press. Deuxième édition.

Raymond, E. dir. (2011), Vox Poetae. Manifestations auctoriales dans l'épopée gréco-latine, CEROR, Paris.

Rosati, G. (2001), « Mito e potere nell'epica di Ovidio », MD 46, 39-61.

Tarrant, R. (2002), « Ovid and ancient literary history », dans Hardie 2002, 13-33

Wheeler, St. (1995a), « Ovid's Use of Lucretius in Metamorphoses 1.67-68 », CQ 45, p. 200-203.

Wheeler, St. (1995b), «Imago Mundi: Another View of the Creation in Ovid's Metamorphoses », AJP $116,95-121$.

Wheeler, St. (1999), A Discourse of Wonders, Audience and Performance in Ovid's Metamorphoses, University of Pennsylvania Press, Philadelphia. 